\title{
Incremental Validity of Montreal Cognitive Assessment Index Scores in Mild Cognitive Impairment and Alzheimer Disease
}

\author{
Felicia C. Goldstein Aaron Milloy \\ David W. Loring for the Alzheimer's Disease Neuroimaging Initiative \\ Neuropsychology Program, Department of Neurology, Emory University School of \\ Medicine, Atlanta, GA, USA
}

\section{Keywords}

Montreal Cognitive Assessment · Montreal Cognitive Assessment index scores · Incremental validity $\cdot$ Mild cognitive impairment . Alzheimer disease

\begin{abstract}
Background/Aims: The aim of this paper was to evaluate the incremental validity of the Montreal Cognitive Assessment (MoCA) index scores and the MoCA total score in differentiating individuals with normal cognition versus mild cognitive impairment $(\mathrm{MCl})$ or Alzheimer disease (AD). Methods: Effect sizes were calculated for Alzheimer's Disease Neuroimaging Initiative research participants with normal cognition $(n=295), \mathrm{MCl}(n=471)$, or AD $(n=150)$. $\boldsymbol{R e}$ sults: Effect sizes for the total score were large $(>0.80)$ and exceeded the index scores in differentiating those with $\mathrm{MCl}$ versus normal cognition, $M C l$ versus $A D$, and $A D$ versus normal cognition. A combined score incorporating the Memory, Executive, and Orientation indexes also improved incremental validity for all 3 group comparisons. Conclusion: Administration of the entire MoCA is more informative than the index scores, especially in distinguishing normal cognition versus $\mathrm{MCl}$. A combined score has stronger incremental validity than the individual index scores.

(c) 2018 S. Karger AG, Basel
\end{abstract}


Goldstein et al.: Incremental Validity of MoCA Index Scores

\section{Introduction}

The Montreal Cognitive Assessment [1] (MoCA) is a popular test used for cognitive screening in dementia clinics. Traditional interpretation of the MoCA is based upon the summed points across all items, with a maximum of 30 points. Recently, Julayanont et al. [2] developed MoCA index scores for the domains of memory, executive function, visuospatial function, language, attention, and orientation. The investigators found that both the traditional total score and the Memory Index Score, consisting of the performance on delayed free, cued, and recognition conditions of the word list, were strong predictors of conversion from mild cognitive impairment (MCI) to Alzheimer disease (AD) over an average follow-up of 18 months. Conversion to AD was $90.5 \%$ in patients with scores below the cutoff for impairment on both the total score ( $<20 / 30$ points) and the Memory Index Score $(<7 / 15$ points), $74.5 \%$ when 1 score was below the cutoff, and $53 \%$ when both scores were above the cutoff. While not as strong as the Memory Index (area under the curve $[A U C]=0.66$ ) in predicting conversion to $\mathrm{AD}$, Julaynont et al. [2] reported that with the exception of the Language Index Score $(A U C=0.58)$, the AUCs of the other index scores (range $=0.61-0.63$ ) were also significant predictors of conversion from MCI to AD.

In the current study, we investigated the incremental validity of the MoCA index scores versus the MoCA total score in differentiating between cognitively normal individuals and those with MCI or AD. Our goal was to determine the clinical utility of domain-based scoring [2] by calculating effect sizes. Effect sizes reflect the strength of statistically significant differences in performance between the groups and can provide a metric for comparing the diagnostic utility of various measures. We expected that raw scores of the derived index measures would significantly differ among the 3 groups of participants (cognitively normal $>\mathrm{MCI}>\mathrm{AD}$ ) and that there would be differences in the strength of the effect sizes.

\section{Methods}

\section{Participants}

Data used in the preparation of this article were obtained from the Alzheimer's Disease Neuroimaging Initiative (ADNI) database (adni.loni.usc.edu). The ADNI was launched in 2003 as a public-private partnership, led by the principal investigator Michael W. Weiner, MD. The primary goal of ADNI has been to test whether serial magnetic resonance imaging, positron emission tomography, other biological markers, and clinical and neuropsychological assessment can be combined to measure the progression of $\mathrm{MCI}$ and early AD. Inclusion criteria for ADNI require an age range between 55 and 90 years, a minimum of 6 years of formal education, fluency in English or Spanish, Hachinski Ischemic Scale [3] scores $\leq 4$ points (out of a possible 18), and Geriatric Depression Scale [4] Short Form scores $<6$ points (out of a possible 15). Subjects are excluded if they were taking any medications with anticholinergic properties or if they regularly used narcotic analgesics ( $>2$ doses per week). Additional inclusion/exclusion criteria are available at http://www.adni-info. org/Scientists/ADNIStudyProcedures.aspx.

Diagnosis of cognitively intact, amnestic MCI, or AD in ADNI is based on a cognitive screening battery that includes the Mini-Mental State Examination (MMSE) [5], immediate and delayed recall of the first Logical Memory story (Anna Thompson) from the Wechsler Memory Scale-Revised [6], and the Clinical Dementia Rating (CDR) interview [7] conducted with each subject and their program partner. Based upon performance guidelines in the ADNI protocol, controls are defined as having no significant memory complaints beyond those expected for age, a specified education-adjusted cutoff score on Logical Memory delayed recall, a MMSE score between 24 and 30 points, a CDR score of 0 (including a 0 on the Memory Box score), and intact instrumental activities of daily living (IADL). MCI subjects have a memory complaint or a memory problem noted by their partner, a specified education-adjusted cutoff score on Logical Memory, a MMSE score between 24 and 30, a CDR score (including the Memory Box score) of 0.5, and relatively preserved IADL. Finally, participants with AD have a memory complaint or memory problem 
noted by their study partner, an education-adjusted cutoff score on Logical Memory delayed recall, a MMSE score between 20 and 26, a CDR score between 0.5 and 1.0, and meet NINCDS/ADRDA [8] criteria for probable AD.

\section{Procedure}

We used the scores from participants' first administration of the MoCA during their baseline study visit. The ADNI database includes item level data, thereby allowing for calculation of a total score and index scores. MoCA scores were converted into 6 index scores based on the item combinations used by Julayanont et al. [2]. The Memory Index Score consisted of the number of words recalled in delayed free, category-cued, and multiple-choice conditions, multiplied by 3, 2, and 1, respectively (0-15 points). The Executive Index Score included Trail-Making, Clock, Digit Span, Letter A Tapping, Serial 7 Subtraction, Letter Fluency, and Abstraction (0-13 points). The Visuospatial Index Score consisted of Cube Copy, Clock, and Naming (0-7 points). The Language Index Score included Naming, Sentence Repetition, and Letter Fluency (0-6 points). The Attention Index Score was comprised of Digit Span, Letter A Tapping, Serial 7 Subtraction, Sentence Repetition, and Words Recalled in Both Immediate Recall Trials (0-18 points). Finally, the Orientation Index Score included all Orientation items (0-6 points).

We also calculated a combined score by summing the raw scores on the Memory, Executive, and Orientation indexes (0-34 points).

\section{Statistical Analyses}

Analyses of variance and $\chi^{2}$ tests were conducted to evaluate the presence of group differences in demographic variables. Analyses of covariance were used to compare the MoCA total score versus each index score in differentiating between the diagnostic groups (cognitively normal vs. MCI; cognitively normal vs. AD; MCI vs. AD). Bonferroni post hoc analyses were performed. Effect sizes (Cohen's $d$ ) were calculated as the difference between the means of 2 diagnostic groups divided by their weighted pooled standard deviations (SD). Effect sizes were judged to be either small (0.2-0.3), medium (0.5), or large ( $>0.8)$ according to recommended conventions $[9,10]$.

\section{Results}

The sample included 916 participants (295 cognitively normal, $471 \mathrm{MCI}$, and $150 \mathrm{AD}$ ). Participants with $\mathrm{MCI}$ were significantly younger (mean age $=71.6, \mathrm{SD}=7.5$ ) than those with normal cognition (mean $=72.9, \mathrm{SD}=6.0$ ) and $\mathrm{AD}$ (mean $=74.7, \mathrm{SD}=8.2 ; p<0.001$ ), although the effect sizes were small (MCI/normal cognition: $d=0.20$; MCI/AD: $d=0.40$ ). The latter two groups did not significantly differ from each other. There was also a significant difference in the years of completed education $(p<0.01)$, with cognitively normal controls achieving higher levels (mean $=16.6, \mathrm{SD}=2.5)$ than both $\mathrm{MCI}($ mean $=16.1, \mathrm{SD}=2.6)$ and $\mathrm{AD}$ (mean = $15.8, \mathrm{SD}=2.7$ ) participants who, in turn, did not differ from each other, although effect sizes again were small (normal cognition/MCI: $d=0.18$; normal cognition/AD: $d=0.32$ ). There was a smaller percentage of female participants in the cognitively normal group (45.1\%) than in the MCI (54.6\%) and AD (58.7\%) groups ( $p<0.01)$. All 3 groups had comparable distributions of race, with Whites being most often represented ( $>90 \% ; p=0.42$ ).

Analyses of covariance controlling for age, education, and gender revealed significant differences $(p<0.001)$ among the groups in total MoCA scores as well as for each index score. Table 1 shows the demographically unadjusted means and SDs for the groups. Post hoc Bonferroni tests indicated that for all comparisons, the scores of the cognitively normal participants were higher than the scores of both the $\mathrm{MCI}$ and $\mathrm{AD}$ groups. In turn, the MCI participants consistently outperformed the AD participants.

The effect sizes for the MoCA total score and for the index scores are displayed in Table 2. As seen, the effect sizes of the MoCA total score were large across all 3 group comparisons. In addition, these values were larger than the effect sizes of the index scores for every group comparison. For the $\mathrm{MCI} / \mathrm{AD}$ and $\mathrm{AD} /$ cognitively normal group comparisons, most index 
Table 1. Means, SDs, and ranges for the Montreal Cognitive Assessment total score and index scores

\begin{tabular}{lccc}
\hline Measure (max. score) & $\begin{array}{l}\text { Normal cognition } \\
(n=295)\end{array}$ & $\begin{array}{l}\text { MCI } \\
(n=471)\end{array}$ & $\begin{array}{l}\mathrm{AD} \\
(n=150)\end{array}$ \\
\hline Total score (30) & $25.62 \pm 2.51,16-30$ & $23.23 \pm 3.22,13-30$ & $16.93 \pm 4.51,4-25$ \\
Memory Index (15) & $10.44 \pm 3.52,0-15$ & $7.56 \pm 3.86,0-15$ & $4.06 \pm 2.43,0-13$ \\
Executive Index (13) & $11.98 \pm 1.20,8-13$ & $11.24 \pm 1.65,5-13$ & $8.71 \pm 2.89,1-13$ \\
Visuospatial Index (7) & $6.28 \pm 0.90,2-7$ & $6.01 \pm 1.00,0-7$ & $4.81 \pm 1.48,0-7$ \\
Language Index (6) & $5.45 \pm 0.74,2-6$ & $5.20 \pm 0.96,1-6$ & $4.18 \pm 1.54,0-6$ \\
Attention Index (18) & $16.96 \pm 1.36,7-18$ & $16.23 \pm 1.90,8-18$ & $13.51 \pm 3.39,3-18$ \\
Orientation Index (6) & $5.94 \pm 0.25,4-6$ & $5.67 \pm 0.62,0-6$ & $4.00 \pm 1.52,0-6$ \\
\hline
\end{tabular}

Values are means \pm standard deviations (SD) and ranges. $p$ values are $<0.001$ for all scores; all post hoc Sheffé tests indicate significant group differences (normal cognition $>$ MCI $>$ AD, $p<0.001$ ). MCI, mild cognitive impairment; AD, Alzheimer disease.

Table 2. Effect sizes (Cohen's $d$ ) comparing diagnostic groups on the Montreal Cognitive Assessment total score and index scores

\begin{tabular}{llll}
\hline Measure & $\begin{array}{l}\text { MCI vs. } \\
\text { normal cognition }\end{array}$ & $\begin{array}{l}\text { MCI vs. } \\
\text { AD }\end{array}$ & $\begin{array}{l}\text { AD vs. } \\
\text { normal cognition }\end{array}$ \\
\hline Total score & 0.83 & 1.61 & 2.38 \\
Memory Index & 0.78 & 1.09 & 2.11 \\
Executive Index & 0.51 & 1.08 & 1.48 \\
Visuospatial Index & 0.28 & 0.95 & 1.20 \\
Language Index & 0.29 & 0.79 & 1.05 \\
Attention Index & 0.44 & 0.99 & 1.34 \\
Orientation Index & 0.57 & 1.44 & 1.78 \\
Memory, executive, and orientation & & & \\
$\quad$ Indexes combined & 0.91 & 1.61 & 2.65 \\
\hline
\end{tabular}

MCI, mild cognitive impairment; AD, Alzheimer disease.

scores had large effect sizes, with the Language Index for the MCI/AD group comparison approaching a large effect size $(d=0.79)$. In contrast, for the $\mathrm{MCI} /$ cognitively normal group comparison, the effect sizes for the index scores ranged from small (Visuospatial $d=0.28$, Language $d=0.29$, Attention $d=0.44$ ) to medium (Executive $d=0.51$, Orientation $d=0.57$ ) and approached a large effect size for Memory $(d=0.78)$.

\section{Combined Indexes}

Due to the finding that the Memory, Executive, and Orientation indexes had larger effect sizes than the other MoCA indexes, we added the raw scores and calculated new effect sizes to determine if the combined score (out of 34 maximum points) improved diagnostic utility (Table 2, last row). As expected, the combined score in the cognitively normal subjects ( mean $=28.36, \mathrm{SD}=3.80$ ) was significantly higher than in the $\mathrm{MCI}($ mean $=24.45, \mathrm{SD}=4.70)$ and $\mathrm{AD}$ (mean $=16.77, \mathrm{SD}=4.89$ ) groups who, in turn, significantly differed from each other as well $(p<0.001)$. The combined effect size was now large $(d=0.91)$ for the $\mathrm{MCI} /$ cognitively normal comparison, and it exceeded the effect size of the total score $(d=0.83)$. For the MCI/ AD group, the combined effect size $(d=2.65)$ also exceeded the total score $(d=2.38)$. The ef- 
Dementia

Cognitive Disorders \begin{tabular}{l|l}
\hline Dement Geriatr Cogn Disord 2018;45:49-55 \\
\hline DOI: 10.1159/000487131 & $\begin{array}{l}\text { @ 2018 S. Karger AG, Basel } \\
\text { www.karger.com/dem }\end{array}$ \\
\hline
\end{tabular}

Goldstein et al.: Incremental Validity of MoCA Index Scores

Table 3. Diagnostic accuracy of combined scores on the Memory, Executive, and Orientation indexes in detecting mild cognitive impairment or Alzheimer disease versus normal cognition

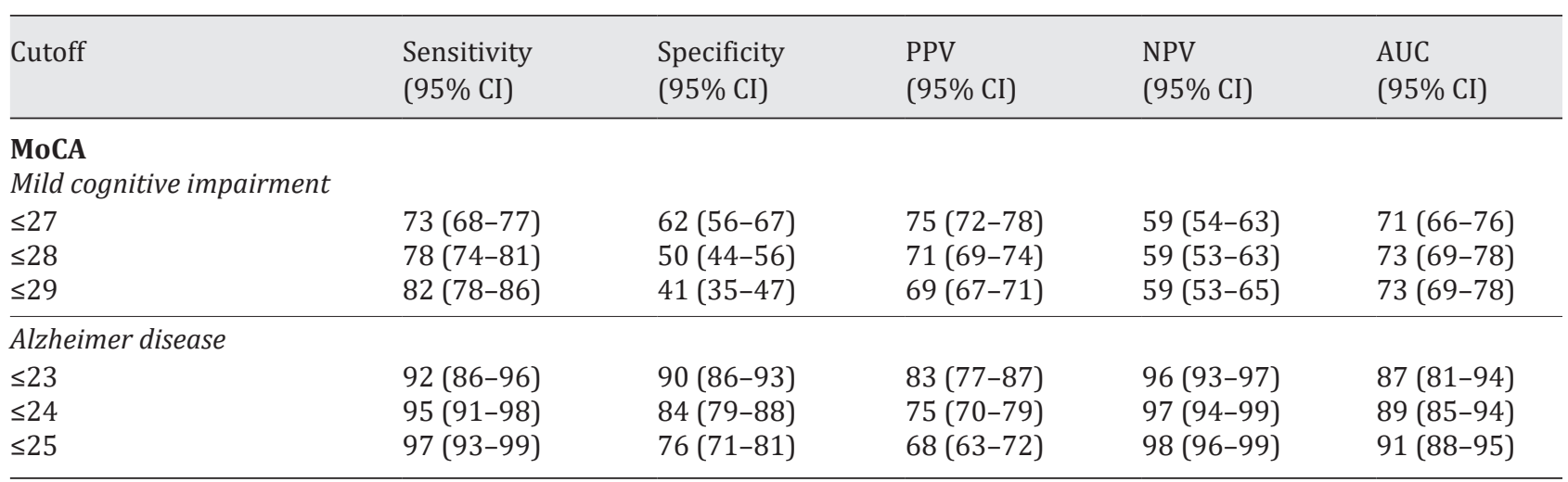

PPV, positive predictive value; NPV, negative predictive value; AUC, area under the curve; CI, confidence interval; MoCA, Montreal Cognitive Assessment.

fect sizes of the total score and combined score were identical for the AD/MCI group comparison $(d=1.61)$.

Table 3 shows the sensitivity, specificity, positive and negative predictive values, and AUCs of various cutoffs in detecting MCI versus normal cognition, and AD versus normal cognition. A combined point value of $\leq 23$ points for the Memory, Executive, and Orientation indexes provided optimal sensitivity (92\%, 95\% CI 86-96) and specificity (90\%, 95\% CI 86-93) for detecting AD versus normal cognition, with 138 of 150 AD patients being correctly classified. A combined point value of $\leq 29$ points yielded reasonable sensitivity $(82 \%, 95 \% \mathrm{CI}$ 78-86) but low specificity (41\%, 95\% CI 35-47) for MCI versus normal cognition, with 388 of 471 MCI patients being correctly classified.

\section{Discussion}

The results of the current study replicate prior findings demonstrating the utility of the MoCA total score as an initial screen for MCI and AD [1,11-14]. The total score had large effect sizes, and these values increased in distinguishing MCI versus cognitively normal $(d=0.83)$, AD versus MCI $(d=1.60)$, and AD versus cognitively normal $(d=2.38)$ groups. The large effect sizes in the current study, based on a research sample screened for MCI and AD, replicates the findings in clinic samples. For example, Larner [12] reported large effect sizes for the MoCA in distinguishing persons with dementia versus no dementia $(d=1.80)$ and MCI versus no dementia $(d=1.45)$. Since the diagnosis of MCI requires evidence of relatively preserved IADL [15], it is not surprising that the MCI group would be hardest to distinguish from those with normal cognition who also have preserved IADL.

Although the point values of the total score and the index scores were significantly different at $p<0.001$ for all comparisons, examination of the effect sizes provided information about the clinical relevance of these statistical differences. These analyses revealed that the effect sizes of the total score were larger than every index score. In addition, while the Memory Index approached a large effect size for the MCI versus cognitively normal groups, the remaining effect sizes ranged from small to medium. In the initial cognitive screening of individuals with subtle deficits, the administration of the entire MoCA may be more informative than the index scores, especially in distinguishing those with normal cognition versus MCI. 
We evaluated whether combining the scores on the MoCA indexes improved incremental validity relative to relying on the separate indexes. We combined the raw scores on the Memory, Executive, and Orientation indexes since individually, these had the highest effect sizes for all 3 groups, and moreover, the items comprising these indexes did not overlap. We found that the effect size of a combined score was larger than their separate index scores, and it was either comparable to the total score (MCI vs. $\mathrm{AD}, d=1.61$ ) or exceeded the effect sizes for the total score (MCI/cognitively normal, $d=0.91$ vs. 0.83 ; AD vs. cognitively normal, 2.65 vs. 2.38). In our study, the combined MoCA score had good sensitivity and specificity for distinguishing AD and normal cognition. However, while sensitivity was high for distinguishing MCI and normal cognition, specificity was weak. The latter indicates that the combined index score is sensitive in detecting cases of MCI but will also incorrectly classify persons with normal cognitive functioning as being impaired. Overall, the results of this investigation suggest that if a person's cognitive status is unknown and the deficits are subtle, such as is often the case in persons with MCI, the administration of the entire MoCA may be prudent. The shortened version combining items from the Memory, Executive, and Orientation indexes may be substituted but will only eliminate 3 items (Naming, Sentence Repetition, and Cube Copy), thus saving a few minutes of administration time at best.

Limitations of the current study include the evaluation of the MoCA index scores in a highly selected group of participants in ADNI who have amnestic single-domain or multidomain MCI. It is therefore not surprising that the MoCA items measuring memory and orientation are especially sensitive to cognitive difficulty. Future studies should include a broader representation of MCI subtypes to see if the effect sizes show a different pattern. In addition, our study is limited to an evaluation of the index scores in a group screened for AD as the primary etiology. Future studies should include a variety of etiologies for cognitive impairment such as frontotemporal or vascular dementia. Finally, Julayanont et al. [2] derived the index scores for the purpose of tracking those persons vulnerable to conversion from MCI to AD. This may be the most useful indication for deriving the index scores and should be replicated in other longitudinal samples.

\section{Acknowledgements}

Data used in the preparation of this article were obtained from the Alzheimer's Disease Neuroimaging Initiative (ADNI) database (adni.loni.usc.edu). As such, the investigators within the ADNI contributed to the design and implementation of ADNI and/or provided data but did not participate in the analysis or writing of this report. A complete listing of ADNI investigators can be found at: http://adni.loni.usc.edu/wpcontent/ uploads/how_to_apply/ADNI_Acknowledgement_List.pdf.

Data collection and sharing for this project was funded by the ADNI (National Institutes of Health Grant U01 AG024904). ADNI is funded by the National Institute on Aging, the National Institute of Biomedical Imaging and Bioengineering, and through generous contributions from the following: Alzheimer's Association; Alzheimer's Drug Discovery Foundation; BioClinica, Inc.; Biogen Idec Inc.; Bristol-Myers Squibb Company; Eisai Inc.; Elan Pharmaceuticals, Inc.; Eli Lilly and Company; F. Hoffmann-La Roche Ltd and its affiliated company Genentech, Inc.; GE Healthcare; Innogenetics, N.V.; IXICO Ltd.; Janssen Alzheimer Immunotherapy Research and Development, LLC.; Johnson \& Johnson Pharmaceutical Research and Development LLC.; Medpace, Inc.; Merck \& Co., Inc.; Meso Scale Diagnostics, LLC.; NeuroRx Research; Novartis Pharmaceuticals Corporation; Pfizer Inc.; Piramal Imaging; Servier; Synarc Inc., and Takeda Pharmaceutical Company. The Canadian Institutes of Health Research are providing funds to support ADNI clinical sites in Canada. Private sector contributions are Rev November 7, 2012, facilitated by the Foundation for the National Institutes of Health (www.fnih.org). The grantee organization is the Northern California Institute for Research and Education, and the study is coordinated by the Alzheimer's Disease Cooperative Study at the University of California, San Diego. ADNI data are disseminated by the Laboratory for Neuro Imaging at the University of California, Los Angeles. 
Goldstein et al.: Incremental Validity of MoCA Index Scores

This work was also supported by an NIH Center Grant for the Emory Alzheimer's Disease Research Center (P50 AG025688).

\section{Statement of Ethics}

All participants provided written informed consent using forms approved by the institutional review boards at each ADNI site.

\section{Disclosure Statement}

There are no conflicts of interest to disclose.

\section{References}

1 Nasreddine ZS, Phillips NA, Bedirian V, Charbonneau S, Whitehead V, Collin I, Cummings JL, Chertkow H: The Montreal Cognitive Assessment (MoCA): a brief screening tool for mild cognitive impairment. J Am Geriatr Soc 2005;53:695-699.

-2 Julayanont P, Brousseau M, Chertkow H, Phillips NA, Nasreddine ZS: Montreal Cognitive Assessment Memory Index Score (MoCA-MIS) as a predictor of conversion from mild cognitive impairment to Alzheimer's disease. J Am Geriatr Soc 2014;62:679-684.

-3 Hachinski VC, Iliff LD, Zilhka E, Du Boulay GH, McAllister VL, Marshall J, Russell RW, Symon L: Cerebral blood flow in dementia. Arch Neurol 1975;32:632-637.

4 Sheikh JL, Yesavage JA (eds): Geriatric Depression Scale (GDS): Recent Evidence and Development of a Shorter Version. New York, The Haworth Press, 1986.

-5 Folstein MF, Folstein SE, McHugh PR: "Mini-mental state": a practical method for grading the cognitive state of patients for the clinician. J Psychiatr Res 1975;12:189-198.

6 Wechsler D: Wechsler Memory Scale - Revised manual. San Antonio, The Psychological Corporation, 1987.

7 Morris JC: The Clinical Dementia Rating (CDR): current version and scoring rules. Neurology 1993;43:24122414.

8 McKhann GM, Knopman DS, Chertkow H, Hyman BT, Jack CRJ, Kawas CH, Klunk WE, Koroshetz WJ, Manly JJ, Mayeux R, et al: The diagnosis of dementia due to Alzheimer's disease: recommendations from the National Institute on Aging-Alzheimer's Association workgroups on diagnostic guidelines for Alzheimer's disease. Alzheimers Dement 2011;7:263-269.

-9 Cohen J: A power primer. Psychol Bull 1992;112:155-159.

10 Cohen J: Statistical Power Analysis for the Behavioral Sciences. Hillsdale, Lawrence Erlbaum, 1988.

11 Freitas S, Simoes MR, Alves L, Santana I: Montreal Cognitive Assessment: validation study for mild cognitive impairment and Alzheimer disease. Alzheimer Dis Assoc Disord 2013;27:37-43.

12 Larner AJ: Effect size (Cohen's d) of cognitive screening instruments examined in pragmatic diagnostic accuracy studies. Dement Geriatr Cogn Disord 2014;4:236-241.

13 Larner AJ: Cognitive screening instruments for the diagnosis of mild cognitive impairment. Prog Neurol Psychiatry 2016;20:21-26.

14 Wojtowicz A, Larner AJ: Diagnostic test accuracy of cognitive screeners in older people. Prog Neurol Psychiatry 2017;21:17-21.

15 Winblad B, Palmer K, Kivipelto M, Jelic V, Fratiglioni L, Wahlund LO, Nordberg A, Backman L, Albert M, Almkvist 0 , et al: Mild cognitive impairment - beyond controversies, towards a consensus: report of the International Working Group on Mild Cognitive Impairment. J Intern Med 2004;256:240-246. 